\title{
OPERATOR PREFERENCE OF RETRACTION METHOD DURING ANESTHESIA DELIVERY
}

\author{
Bernadette Alvear $\mathrm{Fa}^{1 \mathrm{a}^{*}}$, Shika Gupta ${ }^{1 \mathrm{~b}}$, Mouchumi Bhattacharyya ${ }^{2 \mathrm{c}}$ \\ 'Department of Integrated Reconstructive Dental Sciences, School of Dentistry University of the Pacific Arthur A. Dugoni \\ San Francisco, CA 94103, USA \\ 2Department of Mathematics, University of the Pacific, Stockton, CA 95211, USA \\ ${ }^{a} D D S$, Assistant Professor, Director of Local Anesthesia \\ ${ }^{b} \mathrm{DDD}$, Assistant Professor \\ cProfessor of Statistics
}

Cite this article:

Alvear Fa B, Gupta S, Bhattacharyya M. Operator preference of retraction method during anesthesia delivery. Stoma Edu J. 2016;3(1-2):10-15.

Introduction: Dental Health Care Providers (DCPs) traditionally use finger retraction or mirror retraction when delivering local anesthesia. Needlestick injuries (NSIs) occur as hand retracting mucosa is likely to experience injury during dental anesthesia delivery. The aim of this paper is to examine a clinical split mouth study comparing two retraction methods and DCP's retraction preference during delivery of dental anesthesia. The clinical implications from this study impact the practitioner's risk of experiencing an NSI.

Methodology: The IRB approved study (clinical trials ID: NCT02414620) compared the comfort and ease of the retraction method used while delivering an anterior superior alveolar (ASA) injection bilaterally. Surveys given to participants asked about comfort and ease of use of retraction methods, as well as preferred method of retraction during anesthesia delivery. Chisquare tests of goodness-of-fit were conducted to investigate whether there was a significant difference in the proportion of respondents that chose the various categories within one criterion. Results: 62 DCPs participated in the study and no reported NSIs. Data from the DCPs report significance ( $p$ value $<0.001$ ) in comfort comparing retraction methods when providing anesthesia. Regarding preference of retraction, 22 prefer mirror, 29 preferred device, and 3 preferred their finger. Regarding ease of retraction used, 30 chose mirror, 18 device, and 10 chose finger.

Conclusion: Our hypothesis and clinical implication were confirmed. More studies need to be conducted regarding the benefits of using a fingerless retraction method and its effectiveness in dental anesthesia. Keywords: dental anesthesia, dental injection technique, dental armamentarium.

\section{Introduction}

Dental Health Care Providers (DCPs) traditionally use finger retraction or mirror retraction when delivering local anesthesia. ${ }^{1-3}$ Existing dental anesthesia curriculums and educational programs have not emphasized techniques other than using the finger for retraction of mucosa. ${ }^{4-7}$ Literature presents cases of needlestick injuries (NSIs) when finger is used to retract mucosa to deliver anesthesia. ${ }^{8,} 9$ Innovations towards dental anesthesia delivery have progressed throughout the years. ${ }^{14,10-13}$ In addition, various instruments exist both in clinical practice and on the market to aid in retraction (cheek retractor, tongue depressor etc). ${ }^{1,14-16}$

In 2010, a device was approved for purchase in the United States for use during dental anesthesia delivery (Fig. 1). This device is a cordless, rechargeable, handheld system that delivers pulsed micro-oscillations to the injection site. The disposable retraction tips consisting of two rubber prongs with an illuminating LED light appropriate for the generation 2 model can be assembled onto the device prior to use. If the DCP applies too much pressure, the device will automatically shut down the oscillating pulses until an appropriate handle and pressure is applied. ${ }^{17}$

The aim of this paper is to examine a clinical split mouth study comparing two retraction methods and DCP's preference during delivery of dental anesthesia. Our hypothesis is that introducing a new device will provide an alternative method to aid in retraction during delivery of dental anesthesia. The clinical implications from this study 


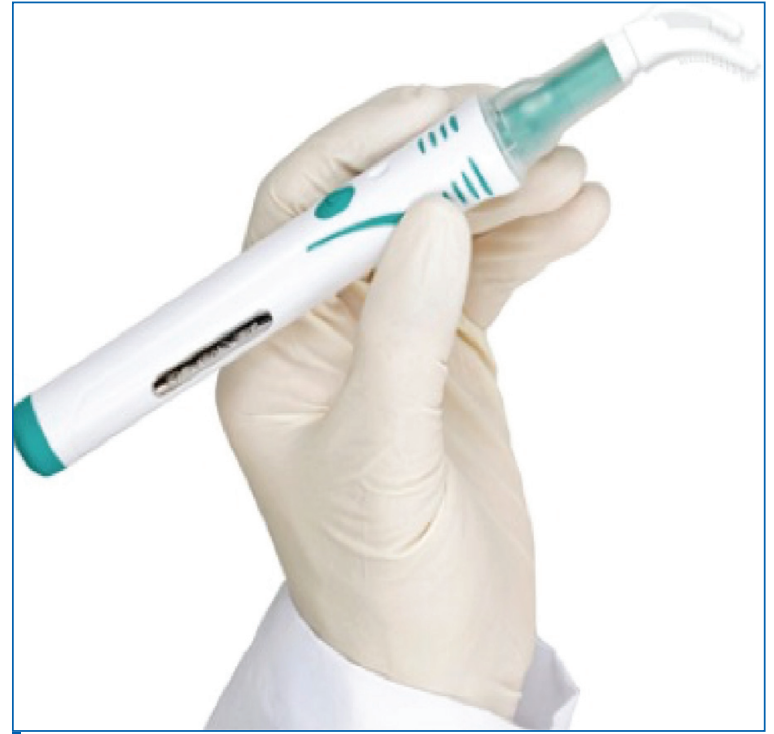

Figure 1. Generation 2 model of Dental Vibe System (device) provided by Bing Innovations, LLC

may impact the practitioner's risk of experiencing an NSI. Unpublished university data have seen preliminary data that relate the reduction in intraoral NSI when a mirror retraction technique was introduced into the curriculum. ${ }^{18}$ Although this paper focusses on the perception of DCPs and their preferred method of retraction, the authors are working on publishing a paper regarding the perception of patients involved in this study.

\section{Materials and Methods}

The IRB approved study (clinical trials ID: NCT02414620) was performed within a university clinical setting, informed consents were obtained from all participants, and occupational safety health administration (OSHA) guidelines were followed. The inclusion criteria for DCPs covered students enrolled in dental and international dental programs, who successfully completed the basic techniques course in their respective local anesthesia curriculum. These novice DCPs initially learned how to provide anesthesia using a dental mouth mirror during their training. Some DCPs also had prior exposure to using their finger as a retraction method to provide dental anesthesia, and none had previous experience using the device. Exclusions from the study covered DCP's who did not complete their basic training in the dental anesthesia curriculum.

The armamentarium used to compare the retraction methods consisted of the mirror and the device (Fig. 1). In addition, a standard dental syringe, 27 gauge short needle, and $1.8 \mathrm{ml} 2 \%$ Lidocaine $\mathrm{HCl}$ 1:100,000 epinephrine were provided. The study protocol compared the comfort of the retraction method used while delivering an anterior superior alveolar (ASA) injection. The participants were required to watch a two minute video describing the proper use of the device. The DCP's were encouraged to ask questions prior to the clinical

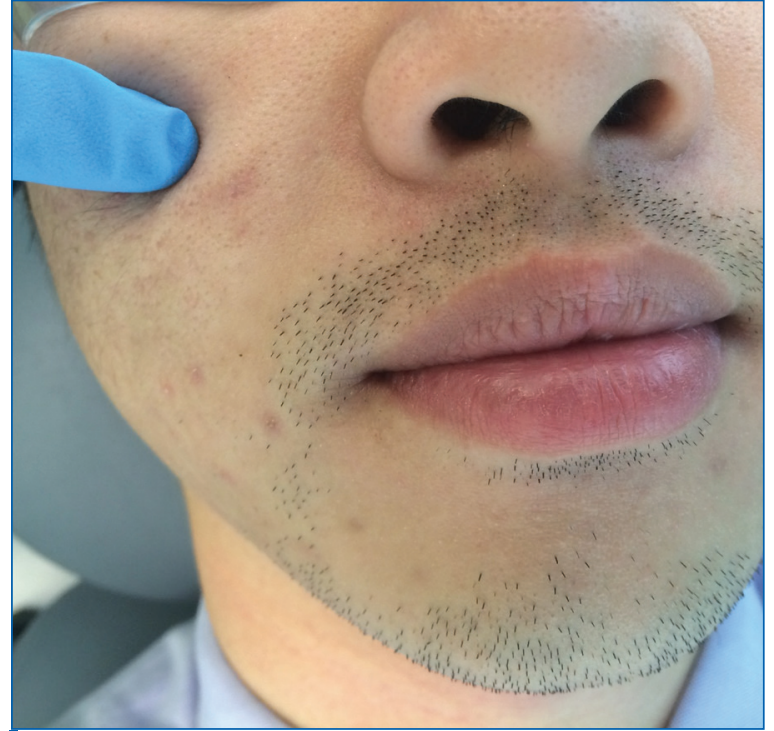

Figure 2. Extraoral palpation of Infraorbital foramen

segment of the study for clarification. The delivery of dental anesthesia was performed under the supervision of the principal investigators.

Prior to the injection, the DCPs palpated for the infraorbital foramen extraorally (Fig. 2). Once the injection sites were identified, a piece of gauze was used to dry a single injection site and then a small amount of topical was placed for about a minute at the insertion site. The insertion site for the ASA is the muccobuccal fold above the maxillary first premolar. ${ }^{1}$ The range of insertion depth was from 5-10 millimeters for each individual patient. The DCPs were required to pull tissue taut with either retraction method applied.

The right side of the mouth used a mirror (control side), while the left side applied the device (device side) as retraction method. After initial penetration into tissue, the needle was slowly advanced apically towards the infraorbital foramen. Once at the deposition site, a supervising investigator verified the depth of insertion and delivery of ASA injection using $0.9 \mathrm{ml}$ of the anesthetic (Fig. 3). Upon reaching the insertion depth, the DCP aspirated during the delivery of the anesthetic to confirm that they were not within a blood vessel and was verified by the supervising investigator.

If aspiration tests were negative, the same syringe and remaining $0.9 \mathrm{ml}$ of anesthetic were administered on the device side, but a new 27 gauge needle was assembled. If aspiration tests were positive, indicated by the entrance of blood into the anesthetic cartridge, then the cartridge and needle were replaced.

Per the manufacturer's training video, the DCPs were instructed to install the disposable tip onto the device in the view of the patient. The DCPs then placed the device over the back part of the patient's hand and communicated to the patient an initial perception of what the device would feel and sound like. The tissue on the device side was 


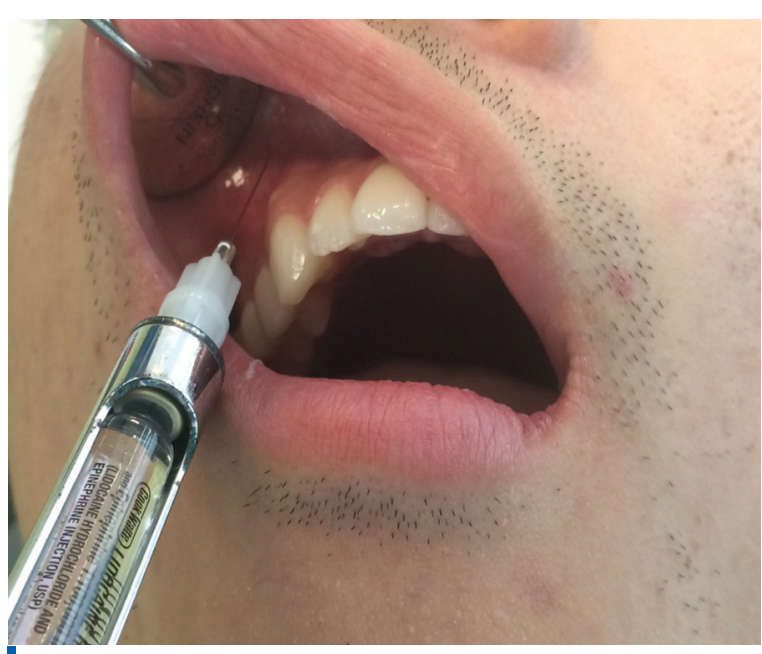

Figure 3. Control side featuring mirror retraction

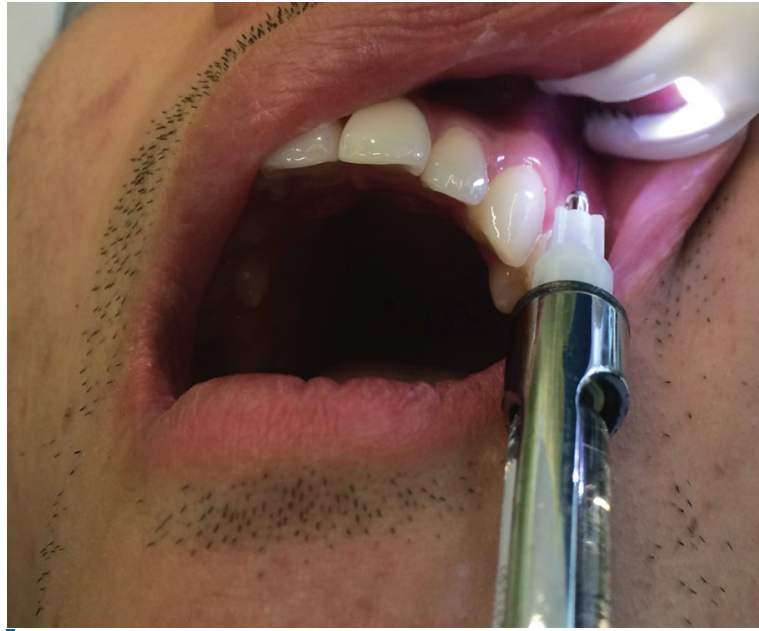

Figure 4. Device side featuring device retraction prepared exactly as on the control side, including topical application after drying the insertion site with $2 \times 2$ gauze. Prior to the injection, the device was placed in the mouth over the area of the insertion site and kept on for five seconds. The needle was inserted in close proximity to one of the two tip prongs, and advanced to the deposition site where anesthetic $(0.9 \mathrm{ml})$ was delivered through an ASA injection (Fig. 4). Verification of insertion depth, amount deposited, and aspiration tests were conducted by both the DCP and the supervising investigator. Upon completion of the delivery of the anesthetic, the needle was removed from the mucosa, and the device remained on for an additional five seconds as instructed by the manufacturer.

The DCPs were given a one page survey (appendix 1) and asked to circle their preferred answer. The questions from the survey inquired about the DCP's perception regarding the study. Question 1 asked if the DCP was compliant with watching the training video for the device. Question 2 asked about anxiousness and comfort when delivering the ASA injection on the control side. Question 3 asked about anxiousness and comfort when delivering the ASA injection on the device side. Question 4 asked the DCPs about their preferred retraction method. Question 5 asked the DCP's which retraction method is perceived as easier.

Chi-square tests of goodness-of-fit were conducted to investigate whether there was a significant difference in the proportion of respondents that chose the various categories within one criterion. In a few surveys, the DCPs chose multiple preferences among the given answers making cross statistical studies meaningless. As a result, these respondents were not part of the analyses, leading to some missing data.

\section{Results}

62 DCPs participated and there were no reported NSIs. The DCP's were compliant with watching the recommended training video from the manufacturer. Regarding comfort and confidence, about the delivery of the anesthesia on the control side, ${ }^{11}$ stated they felt "uncomfortable and anxious" while the remaining 51 responded favorably: "comfortable and confident" leading to a p-value of less than 0.001 . The $p$-value confirms that the proportion of participants who felt comfortable and confident was significantly higher than the proportion that felt uncomfortable and anxious (Table 1).

When asked about comfort and confidence during the delivery of the anesthesia on the device side, 17 DCPs answered they felt "uncomfortable and anxious" while the remaining 45 stated they were "comfortable and confident" leading to a p-value of less than 0.001 (Table 2). As in the previous case, this $\mathrm{p}$-value indicates that the proportion of participants who felt comfortable and confident was significantly higher than the proportion that felt uncomfortable and anxious.

Table 1. During Delivery on Control Side

\begin{tabular}{|c|c|c|}
\hline Comfortable and Confident & Uncomfortable and anxious & p value \\
\hline 51 & 11 & $<0.001$ \\
\hline
\end{tabular}

Table 2. During Delivery on Device Side

\begin{tabular}{|c|c|c|}
\hline Comfortable and Confident & Uncomfortable and anxious & p value \\
\hline 45 & 17 & $<0.001$ \\
\hline
\end{tabular}


The participants were then asked about their preference of the retraction method; 22 responded they prefered the mirror to be used for the delivery of the anesthesia, 29 preferred the device, and 3 preferred their finger as retraction (Table 3). Due to the small number in the last group, it was not possible to conduct a formal statistical analysis.
However, when asked to choose the method that was easier, 30 chose the mirror, 18 the device, and 10 said using their finger for retraction was easier (Table 4). This resulted in a p-value of 0.005 , which indicates that there is a significant difference in the proportions of participants choosing the three different methods.

Table 3. Preference of retraction method

\begin{tabular}{|c|c|c|}
\hline Mirror & Device & Finger \\
\hline 22 & 29 & 3 \\
\hline
\end{tabular}

Table 4. Retraction Method that was easier

\begin{tabular}{|c|c|c|c|}
\hline Mirror & Device & Finger & $p$ value \\
\hline 30 & 18 & 10 & $<0.005$ \\
\hline
\end{tabular}

\section{Discussion}

The results from this study relate with the conclusion from Haskell et. al, that novice practicioners can become comfortable with a learned technique using mirror retraction to deliver anesthesia ${ }^{19}$. From the results, the porportion of participants who felt comfortable and confident, was significantly greater than that of the other porportion feeling uncomfortable and anxious ( $p$-value $<0.001$ ).

In some cases, as with learning how to use a new device, $27 \%$ of the DCP's expressed anxiousness and discomfort. Limited evidence exists assessing anxiousness and discomfort of the DCP when "trying out" novel devices. ${ }^{20}$ Our results coincide with studies stating possible nervousness or fear may exist in some people as there is an introduction of a new stiumuli. ${ }^{19,21-23}$

The results indicate $94 \%$ of the DCPs prefer to use a method of retraction other than their finger for delivery of dental anesthesia. The use of a finger for retraction during anesthesia guides the operator with palpating necessary landmarks prior to the injection, ${ }^{24}$ in addition to the retraction of the mucosa. As mentioned earlier, leaving the finger intraorally during anesthesia delivery puts the retracting hand of the DCP at risk for an NSI. 8,9 Regarding which retraction was easier for the DCP, $83 \%$ of the DCPs stated that the alternative retraction (mirror or device) was easier than using the finger lending to a $\mathrm{p}$ value less than 0.005 among the three methods.

With the many advances and innovations created for the delivery of dental anesthesia, there is a necessity to consider alternative methods of retraction while providing dental anesthesia. ${ }^{9}$ There are reports discouraging DCPs from using their finger for retraction and encouraging DCPs to use a safer method to deliver local anesthesia. ${ }^{13,25,26}$ When changing technique, or working with a new device, DCPs need to review the clinical evaluations to gauge safety ${ }^{27}$ and appropriateness of the innovative devices.

Our hypothesis and clinical implication were confirmed. Comfort and confidence with use of an alternative retraction method (mirror and device) was found to be a constructive perception. The results reassure the profession, not just within a university clinical setting, but also in practice, that there are techniques and armamentarium available to the DCP to assist in retraction during dental anesthesia. Practitioners should consider the value of additional training to get comfortable with an easier and safer technique of delivering anesthesia, ${ }^{28,29}$ especially if their learned technique was the use of a finger retraction. Clinicians may find other devices or armamentarium (tongue depressor, or alternative retractor) that may provide the same retraction method similar to the methods used in this study.

This study provides preliminary data to showcase benefits of using alternative retraction methods. The DCPs participating in this study are considered novice and show preferences and opinions different from DCPs practicing for over 10 years. More studies need to be conducted to further investigate the benefits of using a fingerless retraction method, its relation to NSls, and its effectiveness in dental anesthesia.

\section{Conclusion}

As with providing any dental care, the dental practitioner should be comfortable and confident with the delivery of dental anesthesia. This study explored other techniques for retraction when delivering local anesthesia; an area that needs further exploration. Our results show that noninvasive techniques and armamentarium can be useful when delivering local anesthesia. Retraction techniques help in reducing the risk of NSIs, which is a benefit to the dental providers.

\section{Conflict of interest and financial disclosure}

The authors declare that they have no conflict of interest and there was no external source of funding for the present study.

We would like to acknowledge Bing Innovations, LLC for their support in providing supplies for use with device. 


\section{Appendix 1: Operator Survey for Device Study}

1. Prior to using the device I watched the required training video
a) Yes

b) No

2. When delivering the anterior superior alveolar (ASA) injection using the mouth mirror for retraction, I felt
a) Uncomfortable and Anxious
b) Comfortable and Confident

3. When delivering the anterior superior alveolar (ASA) injection using the device for retraction, I felt
a) Uncomfortable and Anxious

b) Comfortable and Confident

4. The retraction method that I would prefer to use is
a) Mouth mirror
b) Dental Vibe system
c) Finger retraction

5. The retraction method that is easier for me to use is
a) Mouth mirror
b) Dental Vibe system
c) Finger retraction

\section{REFERENCES}

1. Malamed SF. Handbook of local anesthesia. Philadelphia, PA, USA: Elsevier Health Sciences; 2014.

2. Gaum LI, Moon AC. The "ART" mandibular nerve block: a new approach to accomplishing regional anesthesia. J Can Dent Assoc. 1997;63(6):454-459.

3. Meechan JG, Wilson NH. Practical dental local anaesthesia. London: Quintessence; 2002.

4. Callan RS, Caughman F, Budd ML. Injury reports in a dental school: a two-year overview. J Dent Educ. 2006;70(10):10891097.

5. Siew C, Gruninger SE, Miaw CL, Neidle EA. Percutaneous injuries in practicing dentists: a prospective study using a 20 day diary. J Am Dent Assoc. 1995;126(9):1227-1234.

6. Younai FS, Murphy DC, Kotelchuck D. Occupational exposures to blood in a dental teaching environment: results of a tenyear surveillance study. J Dent Educ. 2001;65(5):436-448.

7. Scarlett MI. Local Anesthesia in Today's Dental Practice. dentalcare.com. Continuing Education Course, 2014.

8. Makary MA, Al-Attar A, Holzmueller CG, Sexton JB, Syin D, Gilson MM, Sulkowski MS, Pronovost PJ. Needlestick injuries among surgeons in training. $N$ Engl J Med. 2007;356(26):2693-2699.

9. David HT, Aminzadeh KK, Kae AH, Radomsky SC. Instrument retraction to avoid needle-stick injuries during intraoral local anesthesia. Oral Surg Oral Med Oral Pathol Oral Radiol Endod. 2007;103(3):e11-13.

10. Achar S, Kundu S. Principles of office anesthesia: part I. Infiltrative anesthesia. Am Fam Physician. 2002;66(1):91-94.

11. Joseph, M. Anesthesia Considerations in Dental Practice. In: Urman R, Gross W, Phillip B, editors. Anesthesia Outside of the Operating Room, 1st ed. Oxford: Oxford University Press; 2011

12. Clark TM, Yagiela JA. Advanced techniques and armamentarium for dental local anesthesia. Dent Clin North Am. 2010;54(4):757-768.

13. Scarlett MI. Local Anesthesia in Today's Dental Practice. dentalcare.com. Continuing Education Course, 2014.
14. Siew C, Gruninger SE, Miaw CL, Neidle EA. Percutaneous injuries in practicing dentists: a prospective study using a 20 day diary. J Am Dent Assoc. 1995;126(9):1227-1234.

15. Younai FS, Murphy DC, Kotelchuck D. Occupational exposures to blood in a dental teaching environment: results of a ten-year surveillance study. J Dent Educ. 2001;65(5):436-448.

16. Wray D, Stenhouse D, Lee D, Clark AJE. Textbook of general and oral surgery. Edinburgh: Churchill Livingstone; 2003.

17. Bing Innovations. How Dental Vibe ${ }^{\circledR}$ Works. 2015. [Updated 2016, Mar 1: cited 2016, Mar 1.] Available from http://dentalvibe.com/how-it-works/

18. Alvear Fa B, Cuny E. Preliminary evidence supports modification of retraction technique to prevent needlestick injuries. Anesthesia Progress. 2016; //titan/production/a/anpr/ live_jobs/anpr-63/anpr-63-02/anpr-63-02-05/layouts/anpr63-02-05.3d

19. David HT, Aminzadeh KK, Kae AH, Radomsky SC. Instrument retraction to avoid needle-stick injuries during intraoral local anesthesia. Oral Surg Oral Med Oral Pathol Oral Radiol Endod. .2007;103(3):e11-13.

20. Hochman M, Chiarello D, Hochman CB, Lopatkin R, Pergola $S$. Computerized local anesthetic delivery vs. traditional syringe technique. Subjective pain response. N Y State Dent J. 1996;63(7):24-29.

21. Reed KL, Malamed SF, FonnerAM. Local anesthesia part2: technical considerations. Anesth Prog. 2012;59(3):127-136; quiz 137.

22. Rachman S. The conditioning theory of fear-acquisition: a critical examination. Behav Res Ther. 1977;15(5):375-387.

23. Salgotra V, Agrawal R, Mandal S, Kohli S. New gadgets in local anaesthesia: a review. IOSR J Dent Med Sci. 2014;13:62-66.

24. Goldberg S, Reader A, Drum M, Nusstein J, Beck M. Comparison of the anesthetic efficacy of the conventional inferior alveolar, Gow-Gates, and Vazirani-Akinosi techniques. J Endod. 2008;34(11):1306-1311.

25. Hawkins JM, Moore PA. Local anesthesia: advances in agents and techniques. Dent Clin North Am. 2002;46(4):719-732. 
26. Cleveland JL, Barker LK, Cuny EJ, Panlilio AL. Preventing percutaneous injuries among dental health care personnel; National Surveillance System for Health Care Workers Group. J Am Dent Assoc. 2007;138(2):169-178; quiz 247-248.

27. Cuny E, Fredekind RE, Budenz AW. Dental safety needles' effectiveness: results of a one-year evaluation. J Am Dent Assoc. 2000;131(10):1443-1448.
28. Castella A, Vallino A, Argentero PA, Zotti CM. Preventability of percutaneous injuries in healthcare workers: a year-long survey in Italy. J Hosp Infect. 2003;55(4):290-294.

29. Jayanth ST, Kirupakaran H, Brahmadathan KN, Gnanaraj L, Kang G. Needle stick injuries in a tertiary care hospital. Indian J Med Microbiol. 2009;27(1):44-47.

Bernadette ALVEAR FA

DDS, Assistant Professor, Director of Local Anesthesia Department of Integrated Reconstructive Dental Sciences School of Dentistry, University of the Pacific Arthur A. Dugoni San Francisco CA 94103, USA

Dr. Bernadette Alvear Fa, DDS is an assistant professor in the Department of Integrated Reconstructive Dental Sciences. She is the current Director for the Local Anesthesia Curriculum. She began as an instructor in 2006, then became a clinical instructor at the University of Illinois, Chicago in 2008. She returned to the Pacific as an assistant professor in 2010 and actively practices in the Faculty Dental Service Group.

\section{Questions}

\section{Cases in literature present needlestick injury occurring}

$\square$ a. Only in Oral Surgery;

$\square$ b. When in general practice models;

$\square$ c. When finger is used to retract mucosa;

$\square$ d. Only to dentists and hygienists.

\section{The device used in the study features a}

a. Disposable retraction tip;

$\square$ b. Automatic shut down if too much pressure is applied;

$\square$ c. Cordless unit;

$\square$ d. All of the above.

\section{Fingerless retraction during local anesthesia delivery}

$\square$ a. Is proven to be a superior retraction method;

$\square$ b. Is a difficult technique to learn;

$\square$ c. Helps to reduce risk of intraoral needlestick injury of dental provider;

$\square \mathrm{d}$. Is taught in all dental curriculums.

\section{Prior to delivery of the anterior superior alveolar (ASA) injection block, providers should}

$\square$ a. Identify the extraoral landmark

$\square$ b. Look for edentulous areas within the maxilla;

$\square$ c. Retract tissue while using finger;

$\square \mathrm{d}$. Confirm blood is not in cartridge container. 